\title{
Object Based Media, the IoT and Databox
}

\author{
James A. Colley, Andy Crabtree \\ Mixed Reality Lab, University of Nottingham.firstname.lastname@notttingham.ac.uk
}

Keywords: IoT Media Personal Data Experience

\begin{abstract}
In this paper, we report on the potential for the Internet of Things (IoT) to drive new immersive media experiences. We introduce Object Based Media (OBM) and the Databox platform as a means of driving this turn to the IoT and new media experiences, and describe how the bi-directionality of data between these platforms can be utilised to create new immersive media experiences in the 'living room of the future'. We pay particular attention to the privacy, ethical and legal challenges that arise when trying to create this future, most pertinently in relation to GDPR. Our work finds that the fusion of personal data into new media is near-future feasible, but has the potential to be highly problematic if not approached with compliance and accountability in mind.
\end{abstract}

\section{Introduction}

Both traditional broadcast media and more recently media streaming services (e.g. Netflix and Amazon Prime Video) are based on delivering the same linear programing to everyone, albeit not always at the same time. Whilst this has been wholly successful, the increased availability of personal and IoT data in the home leads us to investigate the possibility of utilising data to affect the delivery of media and create new media experiences. The linear and immutable nature of current media leaves little scope for effect when considering how data might impact and alter a media experience.

OBM is a technology developed by BBC R\&D that allows the content of programmes to change according to the requirements of each individual audience member. OBM packages media assets (video clips, audio, subtitles, etc.) as a collection of objects alongside metadata which describes a variety of ways in which the media objects can be assembled. This enables situationally unique media experiences to be delivered on the basis of the viewer's context, environment and preferences. Thus, OBM marks a radical shift from the traditional broadcast model where everyone receives the same content, as media is 'remixed' on the fly in response to local data-driven stimuli.

The living room of the future is a concept being used to research future immersive living room experiences that connect personal and IoT data with OBM to deliver new media content that is adaptive, bi-directional and personalised. Imagine for example, watching a horror movie that can affect your environment, flickering the lights in your living room and waiting until you move to the edge of your seat before delivering a scare scene where the protagonist's monologue talks directly and personally to you.

Leveraging the IoT and personal data in this way has the potential to create exiting and engaging new media experiences. It also presents significant challenges around privacy, ethics and ultimately trust. If personal data is to be collected or accessed for use in the living room of the future then it must be done so in a way that respects the privacy of the data subjects. It might not be acceptable for example, for a media experience to access the entire browser history from a user's iPad, or to perform a sentiment analysis on the personal messages sent using instant messaging platforms. The challenge here is how a user should interact with the living room of the future so that personal data access can be controlled and made accountable to the user.

The living room itself is a social space, there are therefore issues around exposing personal data in a social environment which have the potential to be morally and ethically questionable. For example, an IoT device which tracks location may well know that a husband visits the pub on his way home from work, three times a week. If this data were to become part of a personalised television show, delivered when the whole family is present then the use of this data might be considered unethical. How ethical the use of this data is, is subject to the social context within which the media is delivered; enabling personal control of data exposure in an accountable way is therefore key to enabling the living room of the future to be delivered in an ethically sound manner.

In addition to the above, using personal and IoT data to drive new media experiences requires that those delivering the experience as data processors be compliant with current data protection legislation. European General Data Protection Legislation (GDPR)[1] comes into effect in May 2018 and marks a game changing shift in how personal data is collected, processed and made accountable; the legislation will apply to any personal data that is collected / processed and can be identifiably traced to a European resident.

Ultimately, the adoption and use of technologies which enable the living room of the future rely upon trust between the occupants of the living room and the technology, platforms and services which deliver the media experience. This trust will be made or broken by the accountability of control that is given to the users of the living room.

Databox provides a means of implementing accountable personal control for the user and compliance with legislation for the content provider [11]. Databox is a platform through 


\section{TRADITIONAL MEDIA}

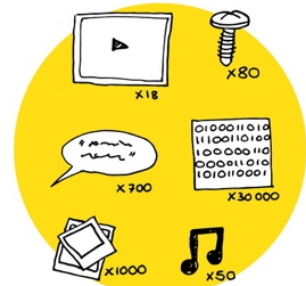

Media is captured using traditional tools.

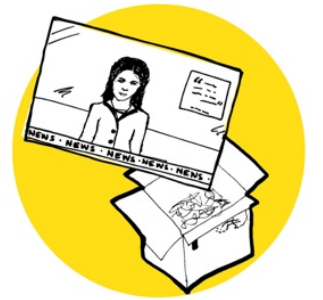

A linear programme is produced from the media.

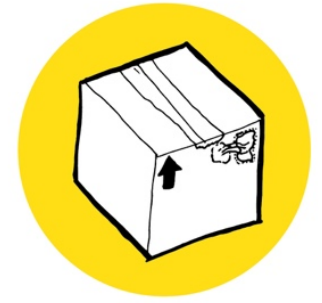

The programme is broadcast to everyone.

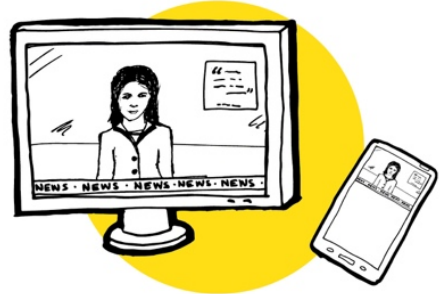

The same content is played back on all devices, resulting in compromises for some experiences.

\section{OBJECT BASED MEDIA}

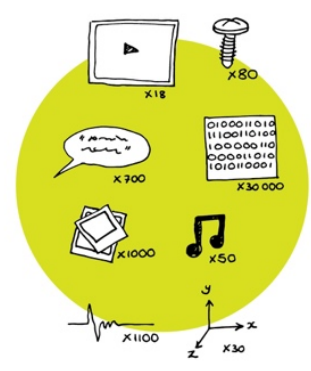

Media is captured using new and traditional tools. Metadata is produced and recorded.

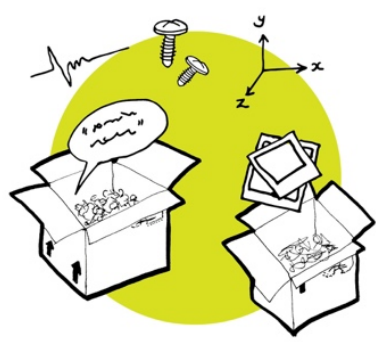

These are packaged as a collection of objects.

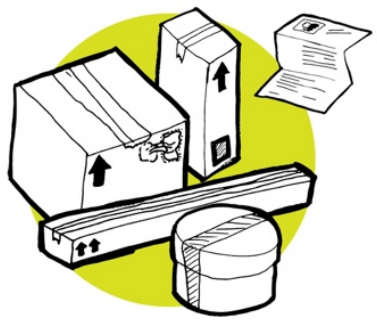

The objects are broadcast to everyone, accompanied by the metadata, which accompanied by the metadata, which
describes all the ways in which the objects can be assembled.

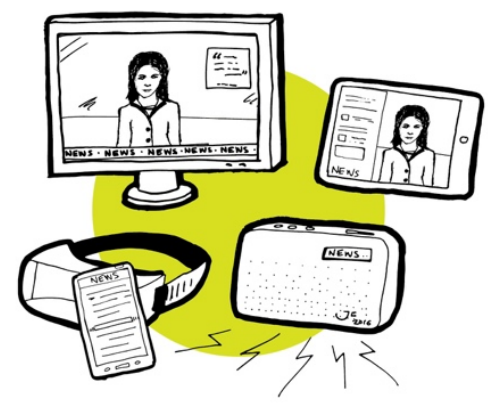

Individual devices in the home assemble objects according to the metadata, producing the best experience for the viewer in the context of their devices, environment, and preference.

$R \& D$

Figure 1 - Object Based Media

which a data subject can make the collection and processing of their personal data accountable to them. It is designed to allow individuals to maintain accountability and control of their personal data whilst still utilising data services and applications that are provided by external parties. Because accountability is implicit within the platform, it also allows data processors to comply with the accountability requirement of GDPR just by using the Databox to deliver their data collection and processing requirements. This paper is a work in progress report of our use of Object Based Media and Databox to deliver, study and understand the challenges around delivering new media experiences within the living room of the future.

\section{The Living Room of the Future}

The living room of the future project is an ongoing collaboration between the British Council, BBC, Foundation for Art and Creative Technology (FACT), and the Mixed Reality Laboratory at the University of Nottingham. The project is working towards creating an exhibition for a future immersive living room experience. This novel experience will explore the relationship between OBM and the IoT, taking advantage of the hyper-connected nature of our homes to provide new media experiences. Within this living room, we will demonstrate how a range of internet connected objects will adapt, interact with and personalise object based media to individuals and groups of people in different ways within the shared space. We aim to show how we can create a personalised, immersive and engaging environment that is both entertaining and educational. This public engagement event and demonstrator is a piece of research that aims to demonstrate and evaluate the concept of IoT augmented experiences through broadcast media, as well as identify the implications of bi-directional, immersive and social broadcast media.

\section{Object Based Media}

Key to delivering the living room of the future experience is Object Based Media (Fig. 1), a BBC R\&D based concept which allows the content of programmes to change according to the requirements of each individual audience member.

The 'objects' refer to the different assets that are used to make a piece of content. These could be large objects: the audio and video used for a scene in a drama - or small objects, like an individual frame of video, a caption, or a signer. By breaking down a piece of media into separate objects, attaching meaning to them, and describing how they can be rearranged, a programme can change to reflect the context of an individual or group of viewers. This approach has potential to transform the way content is created and consumed: bringing efficiencies and creative flexibility to production teams, enabling them to deliver personalised media to every member of the audience. 
The following are example scenarios, taken from the $\mathrm{BBC}$ R\&D website that explain how Object Based Media might form part of everyday life:

My Forecast - "When I watch the weather forecast on iPlayer, I can choose to replace the speaking presenter with a signing one. Because it knows me, iPlayer gives me a signer as default. It syncs with my calendar, knows where I'm planning to go in the next week, and gives me hyper-local forecasts. Ideal for planning my festival wardrobe for Radio 1's Big Weekend!"

Everyone's Wonder of the Universe - "When Wonders of the Universe first started it caused arguments in our house because of the soundtrack. We always loved how atmospheric it is but Gran's hearing issues meant that she had trouble hearing Brian Cox over the music. These days our telly knows when she is in the room and adjusts the audio mix around her so she can hear the dialogue clearly."

EastEnders Catch-up - "I love EastEnders but with four episodes a week there's a lot to catch up on after a fortnight in the sun. iPlayer knows what I've missed and it creates a catch-up episode of Enders just for me. All the juicy bits are there and I'm up to speed in 30 minutes instead of two hours."
It is clear from these examples that the vision of BBC R\&D to use Object Based Media as a more personalised, interactive and immersive way of delivering new media experiences is something that the public may well engage with.

\section{IoT Databox}

In the living room of the future, object based media experiences will need to interact with personal and IoT data; as mentioned in the introduction, this is wrought with privacy, ethical and legal challenges which can be address by using the Databox platform as a way of driving the data requirements of an OBM experience. The Databox is a physical device which acts as a gateway to a distributed platform and is predicated on the 'Dataware model', which sought to develop a business to consumer (B2C) service oriented architecture providing a new wave of personal digital services and applications to individuals [12]. This model posits a user (by or about whom data is created), data sources (e.g., connected devices, which generate data about the user), a personal container (which collates the data produced by data sources and can be accessed via APIs), a catalogue (which allows the user to manage access to the personal container), and data processors (external machines exploited by parties, or "data controllers" in GDPR

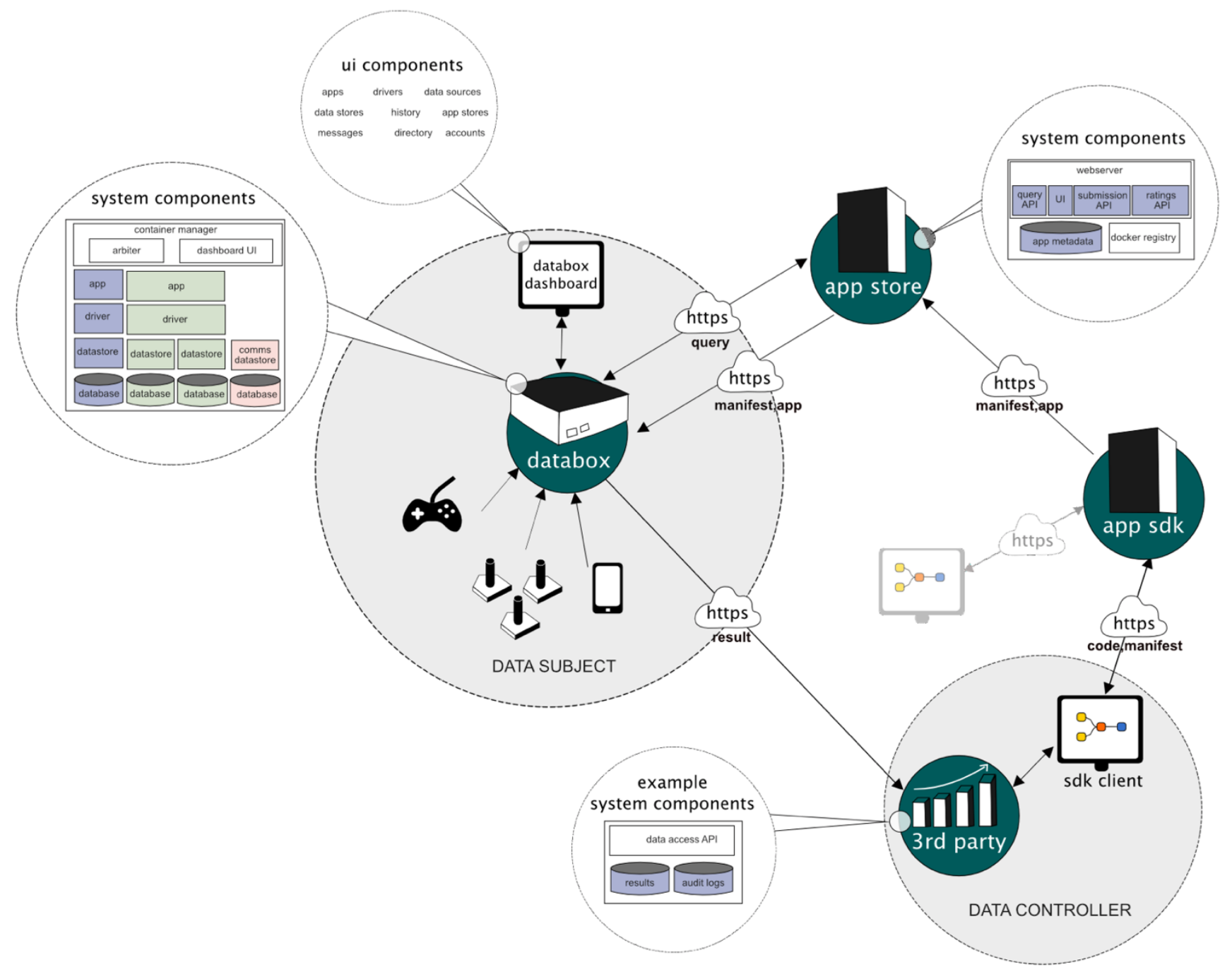

Figure 2 - The IoT Databox Model 
terminology, who wish to make use of the user's data in some way).

The Dataware model is a logical entity formed as a distributed computing system. Data processing involves requests being sent to the catalogue, which are approved or rejected by the user. If approved, the catalogue issues a processing token to the data processor for permitted requests. The processor presents the token to the personal container, which accepts the token, runs the processing request on the relevant data sources, and then returns processed results to the data controller. The Dataware model represents a distinctive approach to personal data processing, that not only seeks to enable user control but also data minimisation. Thus, the Dataware model takes a significant step towards implementing the local control recommendation of the GDPR, minimising data sharing to the results of processing. The raw data remains 'on the box' under the users control.

The Dataware model is currently being reconfigured around the IoT Databox concept, which embeds the Dataware model in a physical object situated in the physical environment (e.g., a networked mini-computer in the home) under the direct control of the individual. It allows the individual to collate data from an array of data sources in a single place and allows the individual to control access to them. Data from individual data sources is stored in 'data stores', i.e., containerised, application-specific, processes [e.g., 4] that reduce the attack surface and management problems associated with general purpose operating systems.

Architecturally the IoT Databox model consists of three key components: the Databox, an app store (of which there may be many), and data controller/third party processors (Fig. 2). The Databox is a small form factor (x86 or ARM) computer consisting of a collection of containerised system services including the dashboard, which provides Databox users with a range of management functions including:

- Creating User Accounts on the Databox and activating sharing permissions (e.g., that consent from all users of shared resources is required for delete actions).

- $\quad$ Adding Data Sources to the box; including assigning ownership to data sources, annotating data sources (e.g., smart plug $\mathrm{X}$ is 'the kettle'), and sharing data sources with other Databox users.

- Configuring Drivers to enable data sources to write to data stores and devices (e.g. lights) to be actuated by Databox apps.

- $\quad$ Managing Data Stores; including sharing stores with other Databox users, and redacting, clearing, or deleting stores.

- Accessing App Stores; apps are recommended by the box based on available data sources but individuals can also search for, download, and rate apps.
- Sharing Apps, with other users within the home and between distributed Databoxes in other homes; the Dashboard also allows apps to be updated and deleted.

- Receiving Notifications; including the results of data processing prior to distribution, sharing requests, app updates, resource contention, etc.

- Auditing data processing operations; including all accesses to data stores, and any data transactions.

The app store is a cloud-based service, interacted with using standard internet protocols (principally HTTPS). It consists of a web server that provides the app store UI supporting human interaction, and a query API providing for programmatic (machine-based) interaction. The app store manages a Docker repository [5] of apps, which are uploaded via the app submission API and indexed by associated metadata.

Using the databox platform to make IoT and personal data use within OBM, allows us to meet the privacy and ethical challenges presented by the living room of the future. Because the access to data and actuation end points is made clear through interactions with the databox dashboard, usage and control of data is made accountable to users who wish to utilise personal and IoT data as part of the living room of the future.

Privacy preservation is key to compliance with GDPR and is required to meet potential ethical concerns of individuals who will experience the living room of the future. A distinct feature of the IoT Databox is that so far as is possible, the data does not leave the box. Data stores remain on the box and applications perform data processing locally and only pass data out of the box with explicit permission of the user.

\section{Designing the living room}

The content for our living room of the future is being developed through a partnership with the British Council. A two day workshop was held in at the Sarajevo Unlimited conference to bring together creatives and technologists and introduce them to the concept of the living of the future. The workshop also acted as a primer to applicants that wanted to apply for a two week residency to create content for the living room of the future exhibition. The workshop introduced the participants to OBM, Databox and helped them to develop proposals for the exhibition residency.

Whilst OBM as a concept is well developed, the authoring of OBM content is still in its infancy. To develop OBM content that can interact with IoT and personal data, we are making use of an OBM engine developed between the Digital Creativity Lab at York University and the BBC. The OBM engine allows authors to drag and drop media content into experience pathways which can diverge, converge and adjust based on input from a 'world context' object. It is this world context object that will act as the point of communication between the OBM engine and the Databox platform. For the Databox to be able to get data into and out of the OBM world context a Databox driver app pair has been developed to provide data to 
the world context and receive requests for actuation from the world context, this provides for bi-directional communication between the OBM engine and databox. Each convergence, divergence and adjustment on the OBM pathway can be affected by personal and IoT data from the databox. IoT devices and personal data set need to have associated Databox drivers in order to get data into the Databox so that the data can be utilised by the OBM engine app. Examples of existing devices that we have built databox drivers are:

- Phillips Hue lights and motion sensors [6] - the driver enables the lights to be switched on or off, and have their colour and brightness set by a Databox app

- Netatmo room sensors [10] - these enable room level sensing of sounds level, carbon dioxide level, ambient temperature and humidity.

- $\quad$ Seat presence sensor - this driver speaks over WiFi to an Adafruit Feather [7] that has pressure mats [8] connected to it. These mats can be placed under sofa cushions to detect when people are sat on the sofa or under floor mats to detect when people walk over them.

- Smart plugs [9] - these can sense the amount of electricity being used by the mains powered device plugged into them, they can also turn the connected device on or off.

As the living room of the future exhibition is developed, it is inevitable that more IoT device and personal data sets will be required to affect the developed OBM pathways, these will require additional development of Databox drivers which will be jointly between the Sarajevo Unlimited residents and the Databox team.

To address the privacy and ethical challenges that personal and IoT data use in object based media presents, the users that take part in the exhibition experience will in some way need to interact with the functions of the Databox dashboard outlined

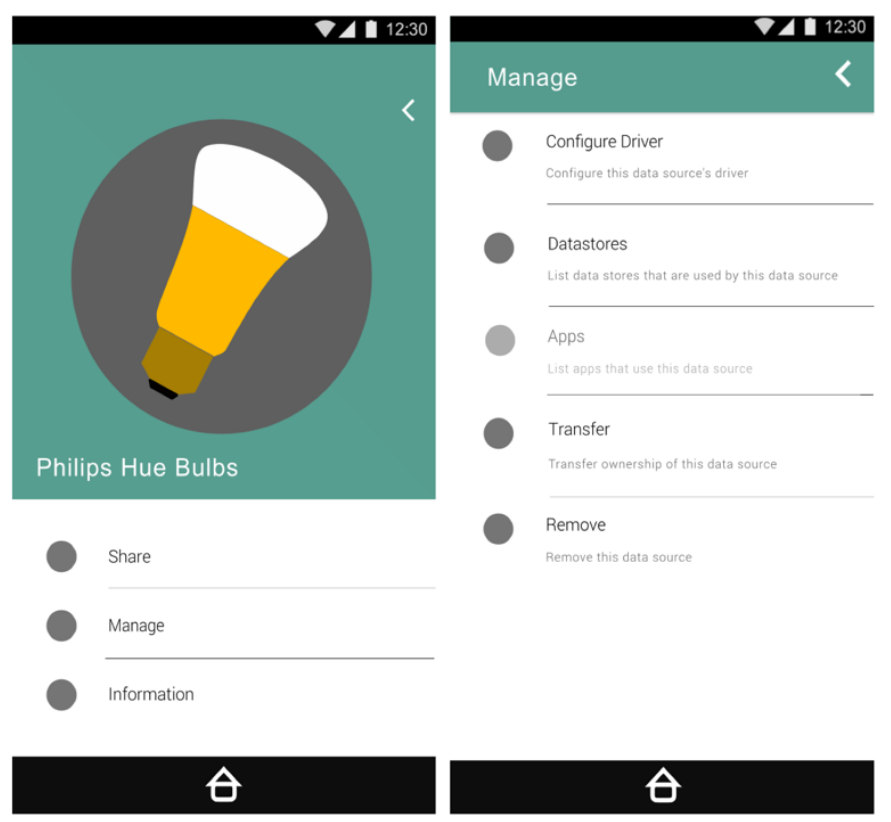

Figure 3 - Databox Dashboard in the previous section. The challenge of how this interaction should take place will be need to be addressed by the OBM content creators in their design of the experience. Whilst the basic and most obvious option would be to have users configure the data permissions using the traditional web based dashboard UI (Fig. 3), the content creators recruited from the Sarajevo Unlimited workshop are being encouraged to come up with new and more engaging ways for users to interact with data access and permissions that might become part of the exhibition experience rather than feel like an adjunct task.

\section{Summary}

We have reported on the work to date in our research into creating new immersive media experiences that utilise personal and IoT using OBM content driven by the IoT databox. We have highlighted the challenges that this new media experiences might face as being ones of privacy, ethics and legal compliance. We have outlined our approach to meeting these challenges using the OBM, OBM engine and the IoT Databox. The living room of the future exhibition will take place at FACT in May 2018 and will be open to the general public.

\section{Acknowledgements}

This work was supported by the British Council [https://kosovo.britishcouncil.org/en/the-living-room-of-thefuture], the Arts and Humanities Research Council [grant number AH/R008728/1], and the Engineering and Physical Sciences Research Council [grant numbers EP/M001636/1 and $\mathrm{EP} / \mathrm{N} 028260 / 2]$

\section{References}

[1] General Data Protection Regulation, in Official Journal of the European Union, vol. 59, pp. 1-88, May 2016.

[2] Consumer Data Privacy in a Networked World: A Framework for Protecting Privacy and Promoting Innovation in the Global Digital Economy, in Journal of Privacy and Confidentiality, vol. 4 (2) , pp. 95-142, March 2013.

[3] Chaudry A., Crowcroft J., Howard H., Madhavapeddy A., Mortier R., Haddadi H. and McAuley, D., "Personal data: thinking inside the box", in Proc. of Critical Alternatives, pp. 29-32, Aarhus, ACM, 2015.

[4] Mudhavapeddy, A. and Scott, D. (2014) "Unikernels: the rise of the virtual library operating system", Communications of the ACM, vol. 57 (1), pp. 61-69.

[5] Docker, www.docker.com [accessed 10-01-18].

[6] Phillips Hue, https://www2.meethue.com [accessed 1001-18]

[7] Adafruit Feather, www.adafruit.com/product/3010 [accessed 10-01-18]

[8] Pressure Mat, uk.rs-online.com/web/p/products/9184756 [accessed 10-01-18] 
[9] TP Link Smart Plugs, http://www.tplink.com/uk/products/details/cat-5258_HS1 10.html [accessed 10-01-18]

[10] Netatmo sensors, https://www.netatmo.com/enGB/product/weather/ [accessed 10-01-18]

[11] Mortier, R., Zhao, J, Crowcroft, J., Li, Q., Wang, L., Haddadi, H., Amar, Y., Crabtree, A., Colley, J., Lodge, T., Brown, A., McAuley, D. and Greenhalgh, C. (2016) "Personal data management with the Databox: what's inside the box?" in Proceedings of the ACM Workshop on Cloud-Assisted Networking, pp. 49-54, Irvine, ACM.

[12] McAuley, D., Mortier, R. and Goulding, J. (2011) "The dataware manifesto", in Proceedings of the $3^{\text {rd }}$ International Conference on Communication Systems and Networks , pp. 1-6, Bangalore, IEEE, 2011. 